\title{
Erratum: Fragile topological insulators protected by rotation symmetry without spin-orbit coupling [Phys. Rev. B 104, 195114 (2021)]
}

\author{
Shingo Kobayashi (i) and Akira Furusaki
}

(Received 16 January 2022; published 26 January 2022)

DOI: 10.1103/PhysRevB.105.039903

We have noticed that Figs. 3(k) and 3(l) in the original published paper are the Wilson loops calculated for the perturbations $\left(\mathcal{M}_{1}^{\prime}, \mathcal{M}_{2}^{\prime}\right)=(0,0.2)$, instead of $\left(\mathcal{M}_{1}^{\prime}, \mathcal{M}_{2}^{\prime}\right)=(0.1,0.08)$ written in the caption of Fig. 3. The corrected figure is shown below, where Figs. 3(k) and 3(1) present the Wilson loop spectra for $\left(\mathcal{M}_{1}^{\prime}, \mathcal{M}_{2}^{\prime}\right)=(0.1,0.08)$. We find in Fig. 3(k) that the Wilson loop eigenvalues for the $C_{4}$-protected index $\bar{v}_{4}$ do not reach the upper and lower limits $\Theta= \pm \pi$, which means $n_{4}^{(-)}(0)=n_{4}^{(-)}(\pi)=0$. In contrast, the $C_{2} T$-protected index $\bar{v}_{2}$ remains stable, as the red (blue) curves in Fig. 3(1) are no longer doubly degenerate but maintain the relative winding number four (zero). Thus, we obtain $\bar{v}_{4}=0$ and $\bar{v}_{2}=4$ for the model Hamiltonian in Eq. (27). The correction does not change our main results. As we have noted in the original published paper, $\bar{v}_{2}$ becomes a $\mathbb{Z}_{2}$ index when the number of occupied bands is greater than two. In any case, the Wilson loop spectra imply that the system is topologically trivial in terms of the $\mathbb{Z}_{2}$ indices $\left(\bar{v}_{2}, \bar{v}_{4}\right)$, while the gapless surface Dirac cones are stable against the perturbations $\left(\mathcal{M}_{1}^{\prime}, \mathcal{M}_{2}^{\prime}\right)$.

Equation (D1) has $V_{4}$, instead of $V_{n}$, due to a typographical error. The corrected equation reads

$$
(-1)^{v_{n, k_{1} k_{2}}}=\exp \left(i \int_{\boldsymbol{k}_{1}}^{\boldsymbol{k}_{2}} d \boldsymbol{k} \cdot \mathcal{A}(\boldsymbol{k})\right) \frac{\operatorname{Pf}\left[V_{n}\left(\boldsymbol{k}_{2}\right)\right]}{\operatorname{Pf}\left[V_{n}\left(\boldsymbol{k}_{1}\right)\right]} .
$$

The caption of Fig. 7 has a typographical error in the perturbation parameters of Fig. 7(e). The corrected ones are written as $\left(\mathcal{M}_{1}, \mathcal{M}_{2}, \mathcal{M}_{1}^{\prime}, \mathcal{M}_{2}^{\prime}, \mathcal{M}_{3}^{\prime}, \mathcal{M}_{4}^{\prime}\right)=(0,0,0,0.03,0.03,0)$. For completeness Fig. 7 is shown below with the corrected caption. 

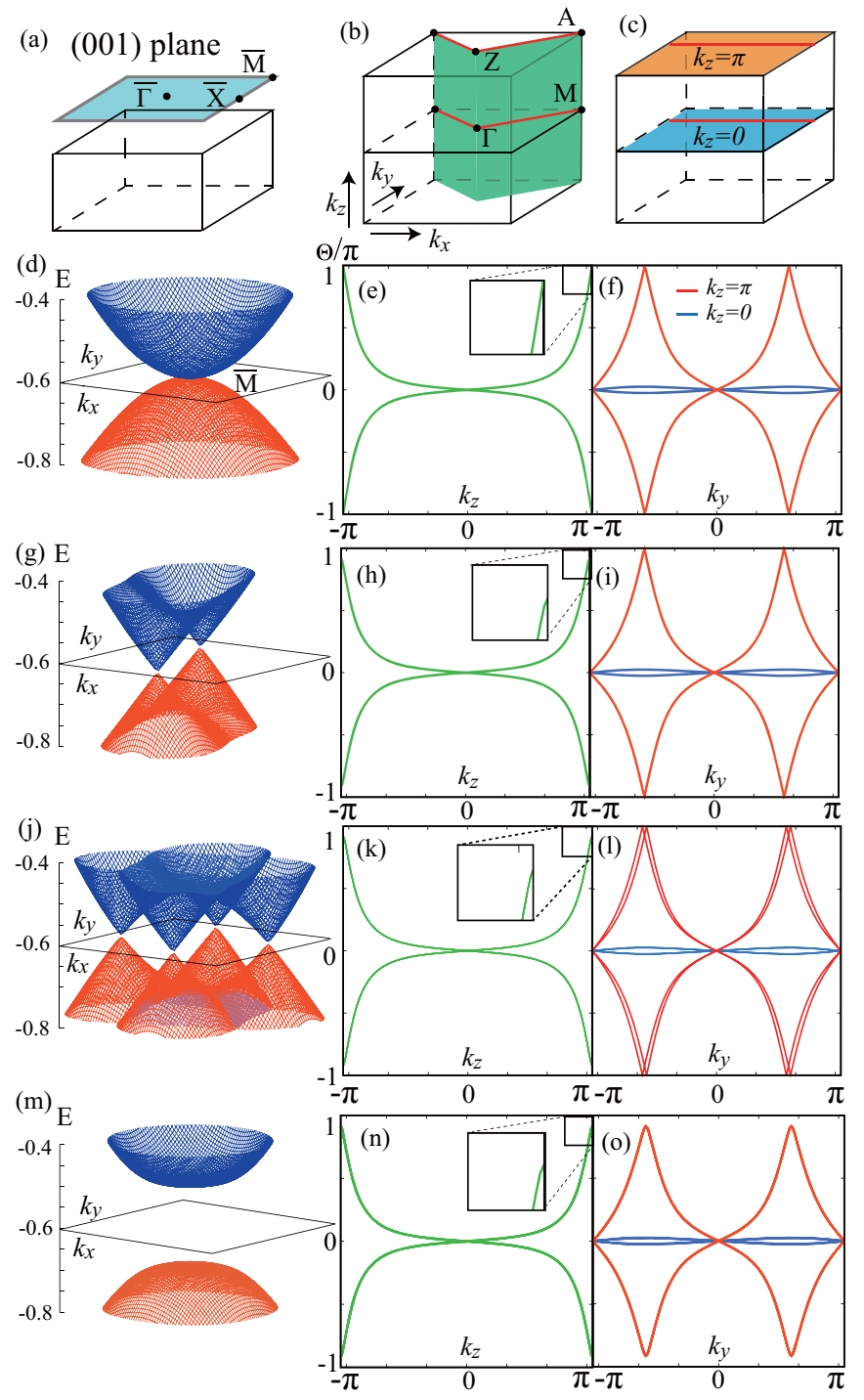

FIG. 3. Surface states and Wilson loop spectra of Hamiltonians in Eqs. (1), (26), (27), and (28), where we choose the same hopping parameters as in Fig. 1 and the perturbation parameters as $\left(\mathcal{M}_{1}, \mathcal{M}_{2}, \mathcal{M}_{1}^{\prime}, \mathcal{M}_{2}^{\prime}, \mathcal{M}_{3}^{\prime}\right)=(0.1,0.08,0.1,0.08,0.1)$. (a) Surface BZ of the (001) plane. (b) The integration path of the Wilson loop operator $\mathcal{W}_{\ell}$ for $C_{4}$ and TR-symmetric insulators. (c) The integration path of $\mathcal{W}_{\ell}$ for $C_{2} T$-symmetric insulators. The surface energy spectra of Hamiltonians in Eqs. (1), (26), (27), and (28) are shown in (d), (g), (j), and (m), respectively. The surface bands are centered around $E=-0.6$. The Wilson loop spectra of the paths depicted in (b) are shown in green as a function of $k_{z}$ in (e), (h), (k), and (n) for Hamiltonians in Eqs. (1), (26), (27), and (28), respectively. The Wilson loop spectra of the paths depicted in (c) are shown in blue $\left(k_{z}=0\right)$ and red $\left(k_{z}=\pi\right)$ as a function of $k_{y}$ in (f), (i), (1), and (o) for Hamiltonians in Eqs. (1), (26), (27), and (28), respectively. 
(a)

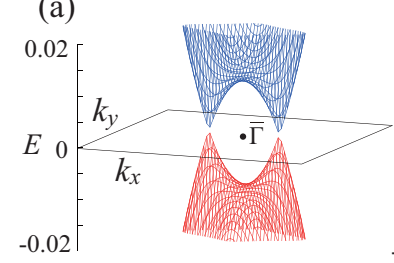

(e)

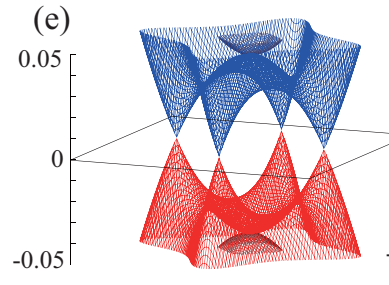

(b)

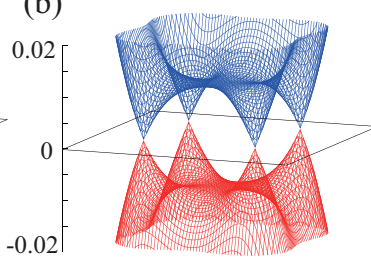

(f) (c)

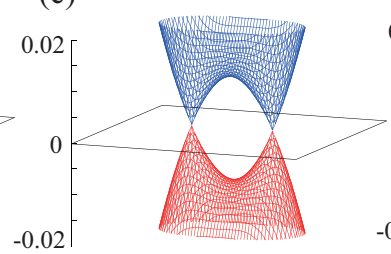

$(\mathrm{g})$ (g)

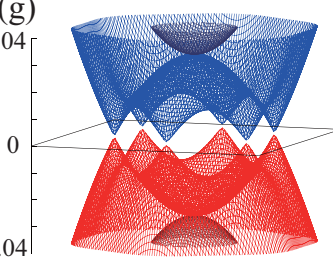

(d)

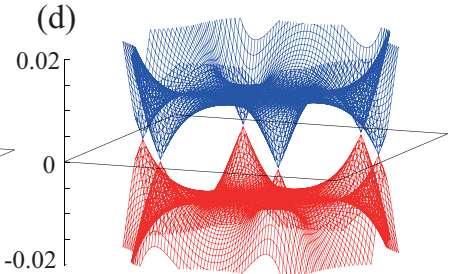

(h)

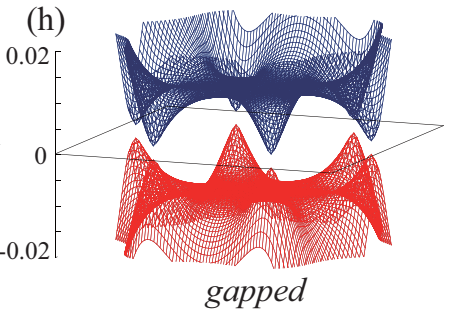

FIG. 7. Surface energy spectra of the tight-binding Hamiltonians, where we choose the same parameters $\left(M_{3}, m_{3}, t, t_{z}\right)=(5,-2,1,1)$. The surface energy spectra of Hamiltonians in Eqs. (F1), (F2), (F3), and (F5) with $\left(\mathcal{M}_{1}, \mathcal{M}_{2}\right)=(0.01,0)$ are shown in (a), (b), (c), and (d). Note that the Hamiltonians in Eqs. (F4) and (F3) have the same surface energy spectra. The surface energy spectra of the double Hamiltonians in Eqs. (F7), (F8), (F9), and (F10) are shown in (e), (f), (g), and (h), where the perturbations are chosen as $\left(\mathcal{M}_{1}, \mathcal{M}_{2}, \mathcal{M}_{1}^{\prime}, \mathcal{M}_{2}^{\prime}, \mathcal{M}_{3}^{\prime}, \mathcal{M}_{4}^{\prime}\right)=$ $(0,0,0,0.03,0.03,0),(0.01,0,0,0.1,0.1,0),(0,0,0,0.03,0.03,0)$, and $(0.01,0,0,0.1,0,0)$, respectively. 